\title{
Philosophy in the Age of Information: A Symposium on Luciano Floridi's The Philosophy of Information
}

\author{
Anthony F. Beavers • Derek Jones
}

Received: 20 September 2013/ Accepted: 27 September 2013/Published online: 11 October 2013

(C) Springer Science+Business Media Dordrecht 2013

This special issue of Minds and Machines contains a number of responses to Luciano Floridi's groundbreaking Philosophy of Information (Oxford 2011). The essays contained here have been grouped by topic; essays 1-5 concern epistemological features of Floridi's approach, and essays 6-8 address his metaphysics.

In "On Floridi's Method of Levels of Abstraction", Jan van Leeuwen addresses Floridi's operational definition of a level of abstraction. Emphasizing the link between Floridi's notion of abstraction and that used in computer science, van Leeuven notes that the method of levels of abstraction may require supplementation in the form of annotation. Annotations, which exist "outside" the formal limits of the levels of abstraction to which they are attached, specify what perspective should be taken towards those levels. Annotations so understood both facilitate the expression of semantic information and elucidate the relationship between levels of abstraction. van Leeuwen then uses a case from the field of hypercomputation to demonstrate that an unbounded number of levels of abstraction can be generated for a system.

In "Information Dynamics", Amos Golan draws connections between Floridi's work with a number of issues in info-metrics. In so doing he addresses the matter of information processing directly and links it to two other open problems identified by Floridi-the problem of how data acquire their meaning and the question of how information can be assessed. Defining information dynamics in terms of a process of transforming input information into output information, Golan addresses the problem of how information processing can occur in cases where input information is incomplete, limited or noisy. Golan presents both a broad definition of

\footnotetext{
A. F. Beavers $(\bowtie) \cdot$ D. Jones

The University of Evansville, Evansville, IN, USA

e-mail: tb2@evansville.edu

D. Jones

e-mail: dj4@evansville.edu
} 
information and a general formulation of information processing, relating both to central themes in Floridi's work.

In "Towards an Informational Pragmatic Realism", Ariel Caticha utilizes the method of entropic inference as a general framework for reasoning in uncertain conditions. Working within a Bayesian framework, he argues that information must be defined in terms of its effects on the beliefs of rational agents. Caticha defines information in terms of constraint on rational belief, identifies pragmatic elements common to Floridi's informational structural realism, Putnam's internal realism and van Fraasen's empiricist structuralism, and proposes an informational pragmatic realism that unifies these elements into a coherent doctrine.

In "Relevant Information and Relevant Questions. Comment on Floridi's 'Understanding Epistemic Relevance'”, Patrick Allo argues that Floridi's formalization of the "basic case" of epistemic relevance contains an error that carries over to later probabilistic formulations of that case. Allo proposes an analysis of relevance that takes into account multiple questions, where the probability of an agent's being interested in the answer to a given question depends upon the agent's interest in the answer to some prior question. This proposal demands renewed focus on the situated character of the agent, for which the demand to maximize benefits and minimize costs may mean something quite different than it does for ideal rational agents with unlimited resources.

In "Intuition, Computation, and Information", Ken Herold attempts to characterize our experience of information objects. Drawing upon the work of Chudnoff, Turing and Floridi, Herold identifies similarities between perception, intuition, computation, and information across the dimensions of cognitive continuity, temporality, and constitution. Herold concludes by noting that these similarities suggest that the philosophy of information may be usefully brought to bear upon other areas of epistemology.

In "Rethinking Construction. On Luciano Floridi's 'Against Digital Ontology",, Mark Bishop and Chryssa Sdrolia highlight the Kantian character of Floridi's arguments against "digital ontology". The authors identify further difficulties for the digital ontologist's application of empirico-mathematical concepts within a philosophical framework, noting that cross-framework applications that do not respect the constraints unique to model-construction within each framework may fail. They then turn to Floridi's argument by thought experiment against the cogency of attempts to determine whether noumenal reality is analogue or digital, arguing that Floridi runs the risk of making an error similar in structure to that made by the proponent of digital ontology.

In "A Revised Attack on Computational Ontology", Nir Fresco and Phillip Staines address the central implication of Floridi's thought experiment, arguing that the fact that digital and analogue features are features of the levels of abstraction that we use to analyze reality does not entail that reality is in itself neither analogue nor digital. Fresco and Staines argue that the view of the universe as a deterministic, irreversible, discrete computational system is inconsistent with the Second Law of Thermodynamics. After presenting further argument against reversible computational views, Fresco and Staines conclude that the computability of the universe does not secure its computationality. 
Finally, in "On the Possibility of Quantum Informational Structural Realism", Terrell Bynum endorses Floridi's Informational Structural Realism, particularly his argument for the existence of dedomena, which Floridi takes to be preconditions for any relations whatsoever and which therefore are constitutive of all possible worlds. Bynum offers an extension of Floridi's theory, specific to the actual world in the form of quantum informational structural realism. Reinterpreting the universe as a rapidly-expanding "foam bubble" of "primordial quantum data", Bynum argues that understanding the fundamental elements of reality as informational allows for ready explanation of otherwise mysterious quantum phenomena such as superpositions, decoherence, entanglement, teleportation and quantum computing.

We have enjoyed the process of preparing this volume, which draws out many of the remaining issues in Floridi's philosophy of information, and we hope that the reader enjoys working through these papers as well. In closing, we wish to thank the authors of the papers, who also acted as reviewers for this volume, and Floridi himself for making this work possible. 